\title{
Ontology-Driven Resource Selecting in the Grid Environments ${ }^{\star}$
}

\author{
Wenju Zhang, Yin Li, Fei Liu, and Fanyuan Ma \\ Shanghai Jiao Tong University, \\ Shanghai, P.R.China, 200030 \\ \{zwj, liyin, liufei, fyma\}@cs.sjtu.edu.cn
}

\begin{abstract}
In the Grid environments where many different implementations are available, the need for semantic matching based on a defined ontology becomes increasingly important. Especially for service or resource discovery and selection. In this paper, we propose a flexible and extensible approach for solving resource discovery and selection in the Grid Environments using ontology and semantic web and grid technologies. We have designed and prototyped an ontology-driven resource discovery and selection framework that exploits ontologies and domain knowledge base. We present results obtained when this framework is applied in the context of drug discovery grid. These results demonstrate the effectiveness of our framework.
\end{abstract}

\section{Introduction}

The need to discover and select entities that match specified requirements arises in many contexts in distributed systems like Peer-to-Peer networks and Grids. In such environments, many different nodes, possibly spanner across multiple organizations, need to share resource 3 .

A common issue both in Peer-to-Peer and Grid is related to the fact that data and resources need to be described in a way that is understandable and usable by the community that is target user, by means of ontologies.

In this paper, we propose a flexible and extensible approach for performing Grid resource discovery and selection using an ontology-driven model and an OMatch resource rank algorithm. Unlike the traditional Grid resource matching that describe resource request properties based on symmetric flat attributes, separate ontologies are created to declaratively describe resources and job requests using an expressive ontology language. Moreover, we propose an O-Match resource rank algorithm to balance the symmetric and asymmetric matching.

The rest of this paper is organized as follows. Section 2 lists the related work. Section 3 presents ontology-driven resource discovery and selection techniques and algorithms. Section 4 presents the prototype implementation and experimental results. Finally, Section 5 gives the conclusions.

\footnotetext{
* This research work is supported in part by the the National High Technology Research and Development Program of China (863 Program), under Grant No. 2004AA104270.
} 


\section{Related Work}

Related to the resource discovery and selection solution. Globus MDS and UDDI are two such examples; MDS has been widely used in the Grid community for resource discovery while UDDI has been used in the web community for business service discovery.

Different approaches for ontology-based resources matching and selecting in the grid systems also have been proposed [2]. Matchmaker 44 is a framework in order to provide a flexible strategy for the resource matching problem in the Grid. This approach is based on three ontologies: a resource ontology, a resource request ontology and a policy ontology.

\section{Ontology-Driven Resource Discovery and Selection}

Semantic matching is based on OWL-S [1] ontologies. The advertisements and requests refer to OWL-S concepts and the associated semantic. By using OWL$\mathrm{S}$, the matching process can perform implications on the subsumption hierarchy leading to the recognition of semantic matches despite their syntactical differences.

\subsection{Ontology-Based Semantic Annotation}

The meaning of services is implicitly expressed by the implementation expressed in the form of the programming language source code. The purpose of the semantic annotation is to express this intrinsic meaning explicitly and in a machine processable way. The Resource Description Framework (RDF) from W3C was designed to serve this purpose and the OWL builds on RDF to provide a way of adding domain specific vocabulary for resource description by using concepts taxonomy.

Semantic annotation of a service is developed in two stages. First, the user annotates a service with intended meaning. Next, different aspects of a service method need to be described independently as distinct resources. This stage concentrates on expressing the syntactic meaning of the service by annotating the semantics of the definition of the service method.

\subsection{Ontology-Based Semantic Matching}

The first step in autonomic service adaptation is to find services that are conceptually equivalent to the client's requirements. These requirements are expressed through the semantic annotation of the interface by using OWL. This ties each of the interface method to a domain concept.

A semantic matching service will need to perform two main inference operations - class and property inferencing. Each interface annotation ties the concept of a method to a ontology class. 


\subsection{The O-Match Algorithm}

In order to enforce dynamic ontology matching, we require a flexible algorithm with the aim of facing two different requirements of the matching process. These requirements have been addressed by the O-Match algorithm for dynamic ontology matching. The aim of O-Match is to allow a dynamic choice of the kind of features to be considered in the matching process. O-Match is based on two basic functions, namely a datatype compatibility function $\mathcal{T}\left(d t, d t^{\prime}\right) \rightarrow\{0,1\}$, and a property and relation closeness function $\mathcal{C}\left(e, e^{\prime}\right) \rightarrow[0,1]$.

The datatype compatibility function $\mathcal{T}\left(d t, d t^{\prime}\right) \rightarrow\{0,1\}$ is defined to evaluate the compatibility of data types of two properties according to a pre-defined set CR of compatibility rules. Given two datatypes $d t$ and $d t^{\prime}$, the function returns 1 if $d t$ and $d t^{\prime}$ are compatible according to CR, and 0 otherwise. For instance, with reference to XML Schema datatypes, examples of compatibility rules that hold between datatypes are: xsd : integer $\Leftrightarrow x s d:$ int, xsd : integer $\Leftrightarrow x s d$ : float, $x s d:$ decimal $\Leftrightarrow x s d:$ floatandxsd: short $\Leftrightarrow x s d:$ int .

The property and relation closeness function $\mathcal{C}\left(e, e^{\prime}\right) \rightarrow[0,1]$ calculates a measure of the distance between two context elements of concepts. $\mathcal{C}\left(e, e^{\prime}\right)$ exploits the weights associated with context elements and returns a value in the range $[0,1]$ proportional to the absolute value of the complement of the difference between the weights associated with the elements. For any pairs of elements $e$ and $e^{\prime}$, the highest value is obtained when weights of $e$ and $e^{\prime}$ coincide.

\section{Prototype Implementation and Experimental Evaluation}

\subsection{Prototype Implementation}

The ontology-based resources discovery and selection framework consists of three components: 1) resources discovery and selection engine, 2) resources database, capturing all the resources available in this domain, and 3) domain ontology knowledge base, capturing the domain model and additional knowledge about the domain.

We have developed two ontologies using OWL-S including resource ontology and domain ontology. The resource ontology provides an abstract model for describing resources, their capabilities and their relationships. The domain ontology is used during the resource selecting process. It is typically defined by the grid middleware.

\subsection{Experimental Results}

To verify the validity of our resources discovery and selection framework and OMatch algorithm, we conducted experiments in the context of the Drug Discovery Grid project's test bed (http://www.ddgrid.ac.cn). which comprised 8 clusters at 5 different cities. We compared the execution time and efficiency of resources discovery and selection with UDDI-based resources selection. 


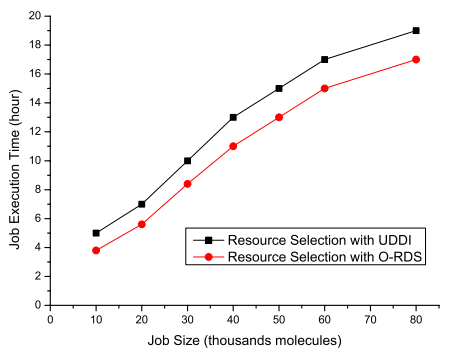

Fig. 1. The job execution time for different job sizes

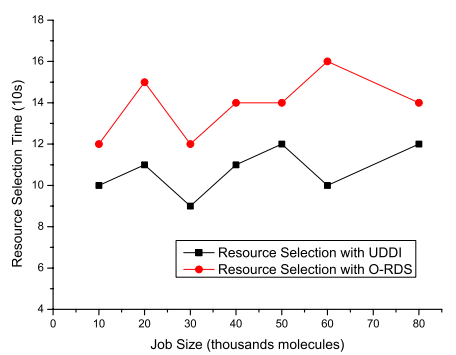

Fig. 2. The resource discovery and selection time for different job sizes

Figure 1 illustrates the job execution time for different job sizes with different resource discovery and selection algorithms. The results show that the job execution time is reduced with the ontology-based resource discovery and selection (O-RDS) algorithm. That is to say, those resources are optimal used at this scenario. Figure 2 shows that the total resource selection time increased with O-RDS algorithm. It is obviously at this dynamically resource selection environment.

\section{Conclusions}

We have proposed a ontology-driven resources discovery and selection framework that provides a common resources selection service for different kinds of application. This framework exploits existing ontology and semantic web and grid technologies. We have used drug discovery grid test bed to validate the design and implementation of the resource selection framework, with promising results.

\section{References}

1. Web Ontology Language (OWL). http://www.w3.org/2004/OWL/.

2. J. Kim, Y. Gil, and M. Spraragen. A Knowledge-Based Approach to Interactive Workflow Composition, Proc. ICAPS workshop Planning and Scheduling for Web and Grid Services, 2004.

3. E. Deelman et al. Mapping Abstract Complex Workflows onto Grid Environments, Journal of Grid Computing, vol. 1, 2003.

4. H. Tangmunarunkit, S. Decker, and C. Kesselman. Ontology-based Resource Matching in the Grid - The Grid Meets the SemanticWeb. In Proceedings of the 1st International Workshop on Semantics in Peer-to-Peer and Grid Computing (SemPGRID) at WWW 2003, pages 706C721, Budapest, Hungary, May 2003. 